\title{
Response to comment on "Hydrochlorothiazide use and the risk of skin cancer in patients with hypertensive disorder: a nationwide retrospective cohort study from Korea"
}

\author{
Eunjung Park ${ }^{1}$, Young Lee ${ }^{2}$, and Mihn-Sook Jue ${ }^{1}$
}

${ }^{1}$ Department of Dermatology, ${ }^{2}$ Veterans Medical Research Institute, Veterans Health Service Medical Center, Seoul, Korea

Received: March 19, 2020 Accepted: March 30, 2020

\section{Correspondence to}

Mihn-Sook Jue, M.D.

Department of Dermatology,

Veterans Health Service Medical

Center, 53 Jinhwangdo-ro 61-gil,

Gangdong-gu, Seoul 05368, Korea

Tel: $+82-2-2225-1388$

Fax: +82-2-2225-4374

E-mail: zooooz@hanmail.net

https://orcid.org/0000-0002-82536188
We thank Drs. Pottegard and Morales for their thoughtful comments on our article entitled "Hydrochlorothiazide use and the risk of skin cancer in patients with hypertensive disorder: a nationwide retrospective cohort study from Korea" [1].

Based on previous studies that hydrochlorothiazide (HCTZ) increases the risk of skin cancer [2,3], and recent recommendations by the European Medicines Agency [4], we conducted this study with the expectation that the use of HCTZ would be associated with an increased risk of skin cancer. Our study showed a much more decreased hazard ratio (HR) in HCTZ-users and this is in contrast to previous other studies reporting HCTZ increased the risk of skin cancer. We also suspected our results differed from previous studies [2,3]. Although not described in the paper, we did an analysis that was adjusted for various types of variables, and did propensity score matching, but the same conclusion was reached that the use of HCTZ significantly reduced the risk of skin cancer. However, time variables to reduce immortal time bias were not used as matching variables. We agree with Pottegard et al. that the start time of follow-up in the design might have induced immortal time bias and subsequently affected the result of our study. Therefore, we are planning to perform reanalysis using Cox regression with time varying exposures. This population-based big data analysis requires cooperation with the Health Insurance Review and Assessment Service (HIRA), and it takes about 6 months to access data, and conduct data preprocessing and data analysis, so it is unfortunate that we cannot provide the results for a newly adjusted immortal time bias analysis as a reply to your comment. Pottegard expressed concern about the bias caused by differences in cumulative HCTZ exposure and duration of follow-up between groups with and without cancer events. However, we performed dose-response tests by classifying exposure to HCTZ into quartiles as well as binary groups of "low users" and "high users" based on a cut-off of 50,000 mg. The mean follow-up period for each quartile dosing group was 5.3, 5.1, 5.6, and 6.7 person years, respectively, showing similar values for each quartile. A clear cumulative dose-response relationship was observed by increasing HCTZ exposure. Even groups with a 1to 2-year cumulative HCTZ duration had a shorter mean follow-up period and less 
than 1 HR when compared to groups with less than 1 year cumulative duration. HCTZ also has a potent and dose-response antioxidative effect as well as photosensitizing effect [5]. Given the above considerations, it is unclear whether the inverse association between HCTZ and skin cancer is only due to immortal time bias. Observational studies are always susceptible to certain limitations and potential biases. Therefore, the results of observational studies alone make it difficult to clarify the association between skin cancer and HCTZ. Further population, race/ethnicity-based epidemiological studies and in vitro studies will be needed to elucidate the association between HCTZ and the risk of skin cancer.

We appreciate your interest in, and comments on our article and we hope that our further analysis and subsequent report will address your concerns.

\section{Conflict of interest}

No potential conflict of interest relevant to this article was reported.

\section{REFERENCES}

1. Park E, Lee Y, Jue MS. Hydrochlorothiazide use and the risk of skin cancer in patients with hypertensive disorder: a nationwide retrospective cohort study from Korea. Korean J Intern Med 2020;35:917-928.

2. Pedersen SA, Gaist D, Schmidt SAJ, Holmich LR, Friis S, Pottegard A. Hydrochlorothiazide use and risk of nonmelanoma skin cancer: a nationwide case-control study from Denmark. J Am Acad Dermatol 2018;78:673-681.e9.

3. Pottegard A, Hallas J, Olesen M, et al. Hydrochlorothiazide use is strongly associated with risk of lip cancer. $\mathrm{J}$ Intern Med 2017;282:322-331.

4. European Medicines Agency. PRAC recommendations on signals [Internet]. London (UK): European Medicines Agency, 2018 [cited 2020 Apr 13]. Available from: https:// www.ema.europa.eu/en/documents/prac-recommendation/prac-recommendations-signals-adopted-3-6-september-2018-prac-meeting_en-o.pdf.

5. Sato K, Dohi Y, Kojima M, Takase H, Suzuki S, Ito S. Antioxidative effects of thiazide diuretics in refractory hypertensive patients. A randomized crossover trial of chlortalidone and trichlormethiazide. Arzneimittelforschung 2010;60:612-616. 